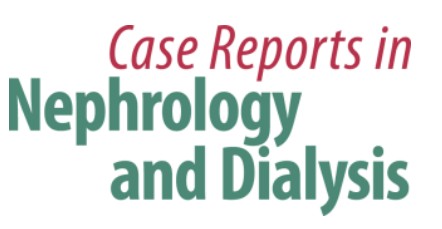

Case Rep Nephrol Dial 2019;9:42-48

DOI: 10.1159/000500105

Published online: April 30, 2019

(C) 2019 The Author(s)

Published by S. Karger AG, Base

www.karger.com/cnd

This article is licensed under the Creative Commons Attribution-NonCommercial 4.0 International License (CC BY-NC) (http://www.karger.com/Services/OpenAccessLicense). Usage and distribution for commercial purposes requires written permission.

\title{
Significance of Crescentic Glomeruli in Acute Kidney Injury with Rheumatoid Arthritis
}

\author{
Ali Ayaash $^{\mathrm{a}} \quad$ Dipesh Maan $^{\mathrm{b}} \quad$ Anastasios Kapetanos $^{\mathrm{e}}$ Mark Bunker $^{\mathrm{c}}$ \\ Mary Chester Wasko ${ }^{d}$ Barbara Clark ${ }^{b}$ \\ aAllegheny General Hospital, Allegheny Health Network, Drexel University School of \\ Medicine, Pittsburgh, PA, USA; 'bivision of Nephrology, Allegheny General Hospital, \\ Allegheny Health Network, Drexel University School of Medicine, Pittsburgh, PA, USA; \\ 'Department of Pathology, Allegheny General Hospital, Allegheny Health Network, Drexel \\ University School of Medicine, Pittsburgh, PA, USA; dDivision of Rheumatology, Allegheny \\ General Hospital, Allegheny Health Network, Drexel University School of Medicine, \\ Pittsburgh, PA, USA; 'Department of Medicine, Allegheny General Hospital, Allegheny \\ Health Network, Drexel University School of Medicine, Pittsburgh, PA, USA
}

\section{Keywords}

Crescentic glomerulonephritis · Acute kidney Injury · Vasculitis · Rheumatoid arthritis · ANCA

\begin{abstract}
Crescentic glomerulonephritis (GN) without immune reactants or deposits (referred to as pauci-immune) is typically characterized by the presence of anti-neutrophilic cytoplasmic antibodies (ANCA). While ANCA-negative patients might be expected to have a more benign course, they often have poor renal outcomes, especially without treatment with steroids and immune-modulating therapy. Pauci-immune crescentic GN can also co-exist with other autoimmune conditions, including rheumatoid arthritis (RA). Here, we describe an ANCA-negative patient with RA who developed dialysis-requiring acute kidney injury (AKI) with findings consistent with focal pauci-immune crescentic GN (i.e., no lgG or immune complex on kidney biopsy). Coexistent conditions included Klebsiella sepsis attributed to pneumonia, rhabdomyolysis, leukocytoclastic immune-mediated skin vasculitis, and positive ANA. He had spontaneous improvement in renal function without immunosuppressive therapy. This crescentic GN was
\end{abstract}




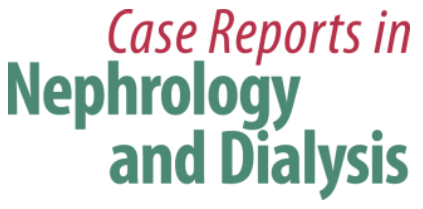

Case Rep Nephrol Dial 2019;9:42-48

DOI: $10.1159 / 000500105$

(c) 2019 The Author(s). Published by S. Karger AG, Basel www.karger.com/cnd

Ayaash et al.: Significance of Crescentic Glomeruli in Acute Kidney Injury with Rheumatoid Arthritis

not associated with poor renal outcome as AKI resolved with supportive care and treatment of his infection. The AKI was likely multifactorial with co-existing acute tubular necrosis in the setting of Kebsiella sepsis and rhabdomyolysis, and the crescentic GN was felt more likely to be related to the infection rather than having a primary role. This case highlights the importance of viewing crescentic GN in the context of the clinical picture, as it may not always lead to the need of aggressive immune suppression and is not a universally poor prognostic kidney finding. However, these cases do warrant close follow-up as our patient had recurrent RA disease manifestations over the next 2 years that eventually led to his death from severe pulmonary hypertension.

(C) 2019 The Author(s)

Published by S. Karger AG, Basel

\section{Introduction}

We present a unique case of a patient with underlying rheumatoid arthritis (RA) with an anti-neutrophilic cytoplasmic antibodies (ANCA)-negative, immune-negative focal crescentic glomerulonephritis (GN) in which the kidney injury was self-limited. Pauci-immune crescentic GN is generally attributed to autoimmune disease characterized histologically by the lack of immunoglobulin deposition within the kidney on immunofluorescent staining. It is the most common cause of rapidly progressive GN in the adult population, with higher rates amongst whites, males, and individuals $>65$ years of age $[1,2]$. The majority of cases of pauci-immune crescentic GN are ANCA positive with ANCA-positive rates reported as high as 80-95\% [1, 3]. However, studies have shown that pauci-immune GN can be ANCA negative in 10-38\% [3-7]. There is still limited research on the characteristics and treatments of ANCA-negative crescentic GN, since these comprise only a small percent of pauci-immune crescentic GN [4-7]. Despite possible different disease processes, the suggested treatments often remain the same with the use of steroids and immune-modulating therapies for most cases [4-7]. Studies that have followed ANCA-negative patients after treatment have found that renal outcomes were either comparable or worse amongst ANCA-negative patients, with the overall survival remaining equal between the two groups [4-7]. There are cases of pauci-immune crescentic GN that co-exist with other autoimmune conditions, including RA, but with uncertain renal significance and variable outcomes [8-12]. An important additional cause of crescentic GN can be infection [13-15]. This case report serves to improve the understanding of the relevance of crescentic GN in RA and the need to be aware of possible infectious triggers rather than autoimmune processes.

\section{Case Presentation}

A 55-year-old male with longstanding RA, hypertension (HTN), and a history of alcohol use disorder was admitted with rash, worsening edema, and abdominal distention for the prior 5 days. He denied fever, chills, sweats, and abdominal pain. He had been treated with methotrexate at RA onset 20 years before but had received no recent RA therapy. There was no history of D-pencillamine use. Additional details of his RA were not available as his medical follow-up had been sporadic. He had joint deformities in his hands but no history of kidney disease or lung disease. There was no radiologic imaging available to determine if there was erosive arthritis. Medications prior to admission included a statin for about 1 year for a history of possible transient ischemic attack and hyperlipidemia and amlodipine for HTN. 


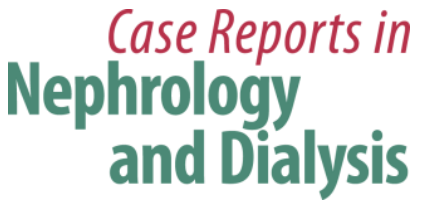

Case Rep Nephrol Dial 2019;9:42-48

DOI: $10.1159 / 000500105$

(c) 2019 The Author(s). Published by S. Karger AG, Basel www.karger.com/cnd

Ayaash et al.: Significance of Crescentic Glomeruli in Acute Kidney Injury with Rheumatoid Arthritis

On physical examination, his temperature was $98.1 \mathrm{~F}$, blood pressure was $142 / 84$, and pulse 104. The patient's heart and lungs were unremarkable, but abdominal examination suggested shifting dullness, and he had marked lower-extremity edema. He had MCP subluxation and ulnar deviation in the hands consistent with RA. A purpuric rash over the lower extremities was noted. Within the first day of hospitalization, the patient developed fever, hypotension, tachycardia, and tachypnea. He was treated with fluid resuscitation, vasopressors, and broad-spectrum antibiotics for suspected sepsis.

Admission laboratory results revealed a hemoglobin level of $9.9 \mathrm{mg} / \mathrm{dL}$, platelets of 81,000, white count of 7.3, AST of $1105 \mathrm{U} / \mathrm{L}$, ALT of $224 \mathrm{U} / \mathrm{L}$, alkaline phosphatase of $426 \mathrm{U} / \mathrm{L}$, total bilirubin 4.6, direct bilirubin 3.6, and creatine phosphokinase of 30,000 U/L. Creatinine was $2.3 \mathrm{mg} / \mathrm{dL}$ (baseline was $0.8 \mathrm{mg} / \mathrm{dL}$ a few months earlier), and urinalysis tested positive for blood on dipstick but no protein or leukocytes. Urine myoglobin returned positive at 1,091 $\mathrm{ng} / \mathrm{mL}$. His statin was discontinued upon admission in the setting of rhabdomyolysis. Initial urine sediment revealed scattered dysmorphic red cells and rare granular casts.

Concern for vasculitis triggered additional workup including serologic testing. This revealed rheumatoid factor 1,512 (normal $<20$ ), cyclic citrullinated peptide $269 \mathrm{U} / \mathrm{mL}$, normal $<17$ ), ANA 1:320, C3 46.8 mg/dL (normal 85-193), and C4 $8.9 \mathrm{mg} / \mathrm{dL}$ (normal 12-36). Testing for MPO-ANCA (myeloperoxidase) and PR3-ANCA (proteinase 3), SSA, SSB, and ds DNA were negative. Hepatitis $B$ and $C$ serologies were negative. Testing for cryoglobulins was negative. Serum electrophoresis was normal, but there was a mild elevation in circulating IgA (409 $\mathrm{mg} / \mathrm{dL}$, normal range 69-382) on quantitative immune globulins. CXR showed multilobar airspace opacities and small bilateral effusions; abdominal ultrasound with a subtle coarse, hepatic echo texture suggesting cirrhosis and mild ascites; as well as normal kidneys and a normal gall bladder.

Initial blood cultures grew Klebsiella oxytoca. CXR revealed progression of bilateral airspace disease and pulmonary edema. Platelet count dropped to 25,000, hemoglobin dropped to $6.1 \mathrm{mg} / \mathrm{dL}$, and white count increased to 19,000 presumed secondary to disseminated intravascular coaguation.

On hospital day 3, he developed oliguria and required continuous veno-venous hemodialysis. After a week of intravenous cefipime and supportive care, all parameters improved, except his kidney function. As he remained dialysis dependent, a kidney biopsy was performed (Fig. 1, 2, 3). Skin biopsy was also performed.

Kidney biopsy was performed, revealing a focal necrotizing GN with a crescent ( 1 of 10 glomeruli, Fig. 1), acute tubular injury (mild) with intratubular myoglobin (Fig. 2, 3). Immunofluorescence was negative, including specific testing for IgG, IgA, IgM, complement C3, and kappa and lambda light chains. Electron microscopy showed no immune deposits. These changes were interpreted by the pathologist as consistent with pauci-immune crescentic GN, acute tubular necrosis, and myoglobin cast nephropathy.

A skin biopsy showed leukocytoclastic vasculitis with positive staining for immunoglobulins IgG, IgA, IgM, and complement C3 (all localizing to the vessel walls).

Treatment with corticosteroids and immunosuppressives for crescentic GN and vasculitis was considered. However, in face of resolving Klebsiella sepsis and otherwise improving clinical status, no immune suppression was given, and the patient was discharged on hemodialysis with plans for close follow-up. His statin medication was not resumed.

Two weeks after discharge, urine output spontaneously returned to normal, and serum creatinine dropped to $1.3 \mathrm{mg} / \mathrm{dL}$. His dialysis was discontinued without incident.

A follow-up creatinine 3 months later returned to his premorbid baseline of $0.8 \mathrm{mg} / \mathrm{dL}$. Follow-up urinalysis at that time was negative for protein or blood, and 24-h protein was 


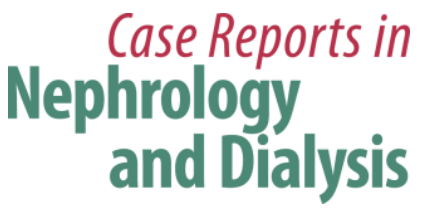

within normal limits at $207 \mathrm{mg} / 24 \mathrm{~h}$. Given the biopsy findings and positive serologies, close follow-up was recommended.

The patient was scheduled for follow-up with rheumatology and nephrology but declined to follow-up as he felt better and lived $2 \mathrm{~h}$ away from the tertiary care hospital.

Communication from his primary care physician several months later noted an episode of inflammatory pericarditis 3 months after discharge that seemed to respond to colchicine and nonsteroidal anti-inflammatory agents. He was asked to follow with cardiology and rheumatology but again declined.

Ten months later, the patient, per primary MD, was noted to have normal kidney function without use of immune-modulating agents. The patient was then lost to follow-up until he presented to an outside hospital 2 years later and was noted to have developed complications of severe right heart failure and pulmonary HTN. He died related to these complications 2 years after his original presentation.

\section{Discussion}

Pauci-immune crescentic GN can co-exist with other autoimmune conditions, including RA [8-12]. However, clinical significance to renal outcome and how aggressively to treat is uncertain. Here, we describe an ANCA-negative patient with RA who developed dialysis-requiring acute kidney injury (AKI) with findings consistent with focal pauci-immune crescentic GN (i.e., no IgG or immune complex on kidney biopsy) as well as ATN. He had spontaneous improvement in renal function without immunosuppressive therapy.

Another consideration in the case presented here was infection-triggered crescentic GN, as infection is also known to trigger crescentic GN and vasculitis and can even be associated with the development of ANCA positivity $[13,14]$. This is particularly the case when the infectious agent is Staphylococcus (which was not the agent in our patient). The patient presented in this case initially had suppressed complement and skin vasculitis with all immune globulins, implying that immune-mediated vasculitis might also be present on kidney biopsy. Most cases of infection-related crescentic GN have immune deposition on immunofluorescence or electron microscopy. Neither of those features was seen in our case. A similar case of pauciimmune ANCA-negative crescentic GN was reported after Gram-negative infection [15]. This was attributed to molecular mimicry related to the homology between the lysosomal protein of the human neutrophil to bacterial adhesion molecule.

Renal involvement related to RA was in the differential diagnosis. Renal disease can occur in RA and may be related to the disease-modifying therapy used to treat the disease in some cases [8-12]. However, when renal disease is present and unrelated to drug toxicity, GN is most likely to be observed. Some studies have reported RA and crescentic GN, but most of these cases were either ANCA positive or failed to report the occurrence of ANCA [8-12]. A case report published in 2003 by Hsieh et al. [12] was the first to describe a case of ANCAnegative pauci-immune crescentic GN in a patient with RA, similar to the patient reported here. The case describes a 37-year-old Chinese female with an established diagnosis of RA who had not been receiving treatment with disease-modifying anti-rheumatic drugs other than herbal Chinese supplements on admission. ANCA was negative. Immunofluorescence of the kidney glomeruli was negative, and a renal biopsy showed crescents in 3/14 glomeruli. The patient was treated initially with IV methylprednisolone and 10 sessions of plasma exchange, which were not adequate since the patient's renal function deteriorated and required maintenance hemodialysis. The study shows that ANCA-negative crescentic GN occurring in 


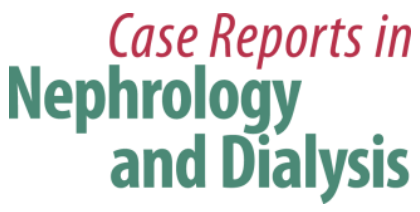

RA can occur and may have severe clinical manifestations. In most cases, treatment of the disease should be considered as soon as crescentic GN is detected to increase the chances of improvement in kidney function.

Despite our patient's complicated history of rheumatological disease (including positive rheumatoid factor, cyclic citrullinated peptide, and ANA), rhabdomyolysis, and leukocytoclastic immune-mediated vasculitis on skin biopsy, the acute renal disease was likely multifactorial, including rhabdomyolysis triggered by Klebsiella sepsis with pneumonia along with statin use as the patient spontaneously recovered renal function, with follow-up urine without any hematuria or proteinuria after treatment of infection and without immune-modulating therapy. It was also deemed likely that the crescentic GN was triggered by the infection and not an autoimmune manifestation.

In these circumstances, with severe AKI, the clinical decision to observe and monitor versus immune suppression can be difficult. In our case, the presence of focal crescent GN on kidney biopsy in a patient with severe AKI was not a harbinger of poor renal outcome. The AKI was felt to be related to his sepsis and rhabdomyolysis. Crescentic GN may be an incidental finding, particularly if focal. If the clinical picture has alternative explanation for the AKI, use of aggressive immune suppression may not always be indicated, particularly as the crescentic GN can be triggered by infection. However, these patients do require close follow-up, as this finding could represent evidence of a smoldering underlying autoimmune process that could lead to adverse outcome in other organs. Our patient did have other subsequent organ system disease with development of symptomatic pericarditis and eventually died 2 years later of complications of severe pulmonary HTN after he had declined close medical follow-up.

\section{Statement of Ethics}

This article does not contain any studies with human participants or animals performed by any of the authors. Informed consent could not be obtained from the patient as he was deceased and next of kin were not reachable. Details of patient information were de-identified. While he had consented to photographs of skin while hospitalized, these were not included in this paper as he had deceased before permission to publish these could be obtained.

\section{Disclosure Statement}

The authors have no conflicts of interest or financial support to declare.

\section{Author Contributions}

All authors participated in the clinical care or diagnostic testing of the patient, assembling of data, and contributed to the intellectual content of the case report, writing of the case report, and actively reviewed and edited the content.

\section{References}

1 Falk RJ. ANCA-associated renal disease. Kidney Int. 1990 Nov;38(5):998-1010.

2 Jennette JC. Rapidly progressive crescentic glomerulonephritis. Kidney Int. 2003 Mar;63(3):1164-77. 


\section{Case Reports in Nephrology and Dialysis}

\begin{tabular}{l|l}
\hline DOI: 10.1159/000500105 & @ 2019 The Author(s). Published by S. Karger AG, Basel \\
\hline
\end{tabular} www.karger.com/cnd

Ayaash et al.: Significance of Crescentic Glomeruli in Acute Kidney Injury with Rheumatoid Arthritis

3 Harris AA, Falk RJ, Jennette JC. Crescentic glomerulonephritis with a paucity of glomerular immunoglobulin localization. Am J Kidney Dis. 1998 Jul;32(1):179-84.

4 Chen M, Yu F, Wang SX, Zou WZ, Zhao MH, Wang HY. Antineutrophil cytoplasmic autoantibody-negative Pauci-immune crescentic glomerulonephritis. J Am Soc Nephrol. 2007 Feb;18(2):599-605.

5 Villacorta J, Diaz-Crespo F, Acevedo M, Guerrero C, Mollejo M, Fernandez-Juarez G. Antineutrophil cytoplasmic antibody negative pauci-immune extracapillary glomerulonephritis. Nephrology (Carlton). 2016 Apr;21(4):301-7.

6 Sharma A, Nada R, Naidu GS, Minz RW, Kohli HS, Sakhuja V, et al. Pauci-immune glomerulonephritis: does negativity of anti-neutrophilic cytoplasmic antibodies matters? Int J Rheum Dis. 2016 Jan;19(1):74-81.

7 Shah S, Havill J, Rahman MH, Geetha D. A historical study of American patients with anti-neutrophil cytoplasmic antibody negative pauci-immune glomerulonephritis. Clin Rheumatol. 2016 Apr;35(4):953-60.

8 Adu D, Berisa F, Howie AJ, Emery P, Bacon PA, McConkey B, et al. Glomerulonephritis in rheumatoid arthritis. Br J Rheumatol. 1993 Nov;32(11):1008-11.

9 Breedveld FC, Valentijn RM, Westedt ML, Weening JJ. Rapidly progressive glomerulonephritis with glomerular crescent formation in rheumatoid arthritis. Clin Rheumatol. 1985 Sep;4(3):353-9.

10 Harper L, Cockwell P, Howie AJ, Michael J, Richards NT, Savage CO, et al. Focal segmental necrotizing glomerulonephritis in rheumatoid arthritis. QJM. 1997 Feb;90(2):125-32.

11 Tebib JG, Trolliet P, Bouvier M, Francois B. Necrotizing glomerulonephritis as a complication of rheumatoid arthritis vasculitis. Br J Rheumatol. 1993 Aug;32(8):765-6.

12 Hsieh HS, Chang CF, Yang AH, Kuo HL, Yang WC, Lin CC. Antineutrophil cytoplasmic antibody-negative pauciimmune crescentic glomerulonephritis associated with rheumatoid arthritis: an unusual case report. Nephrology (Carlton). 2003 Oct;8(5):243-7.

13 Nasr SH, Markowitz GS, Stokes MB, Said SM, Valeri AM, D’Agati VD. Acute postinfectious glomerulonephritis in the modern era: experience with 86 adults and review of the literature. Medicine (Baltimore). 2008 Jan;87(1):21-32.

14 Kallenberg CG, Tadema H. Vasculitis and infections: contribution to the issue of autoimmunity reviews devoted to "autoimmunity and infection". Autoimmun Rev. 2008 Oct;8(1):29-32.

15 Vilayur E. ANCA-negative crescentic GN closely following two episodes of sepsis. Intern Med J. 2015 Mar;45(3):356-7.

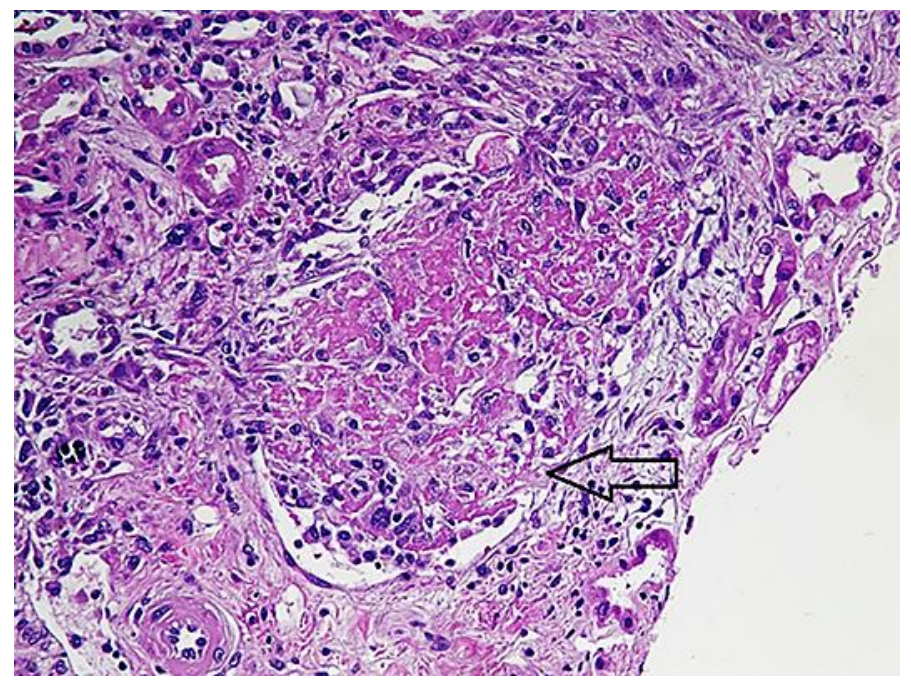

Fig. 1. Hemotoxylin and eosin stain depicting glomerulus advanced necrotizing crescent (arrow). 


\section{Case Reports in Nephrology and Dialysis}

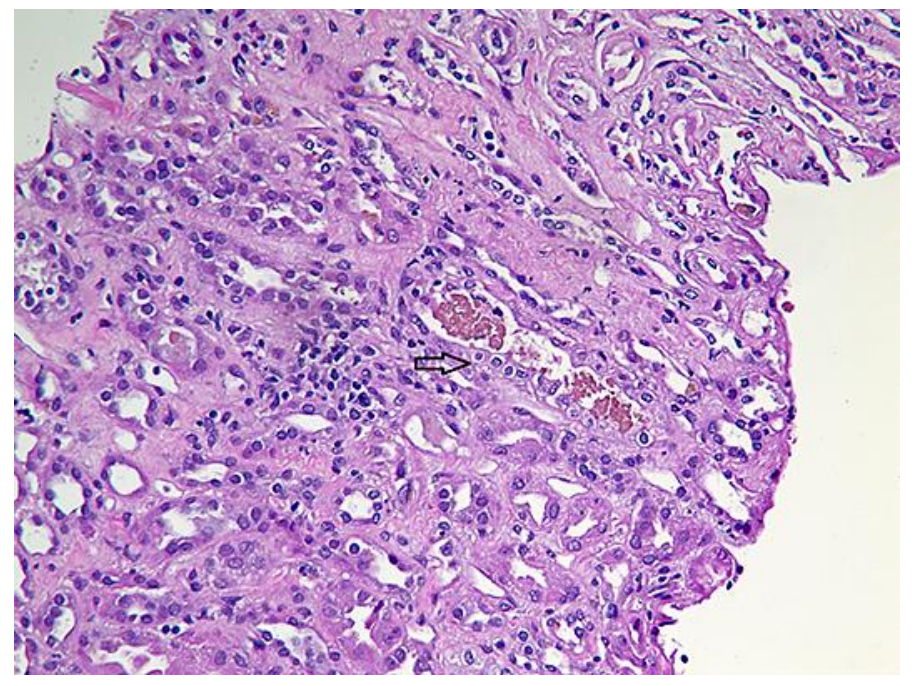

Fig. 2. Hematoxylin and eosin stain depicting intratubular cast (arrow) and tubular necrosis.

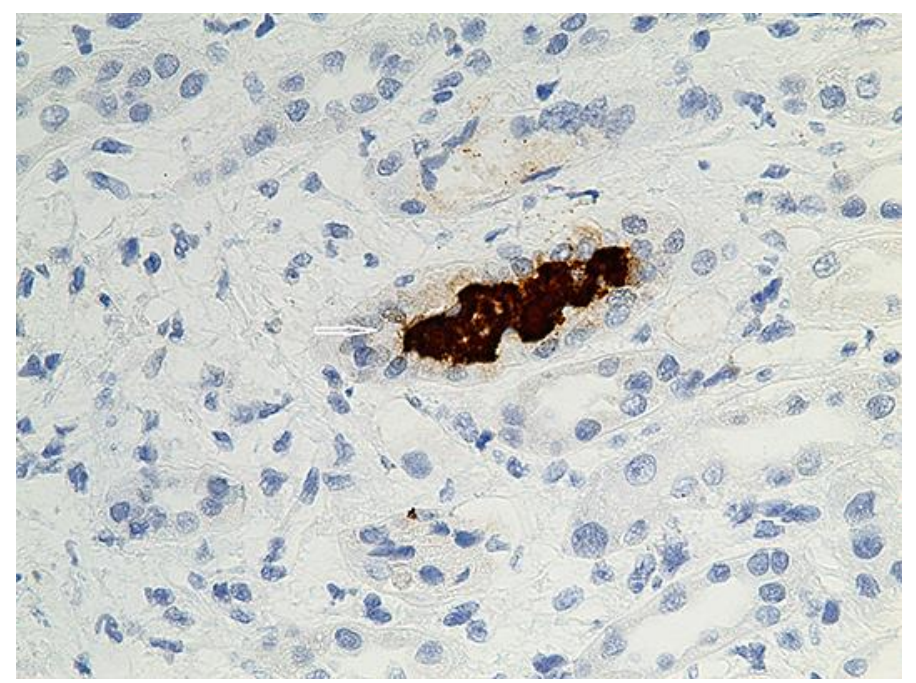

Fig. 3. Intratubular cast stained with myoglobin (arrow). 